\title{
Non-antibiotic methods against Pseudomonas aeruginosa include QS inhibitors: a narrative review
}

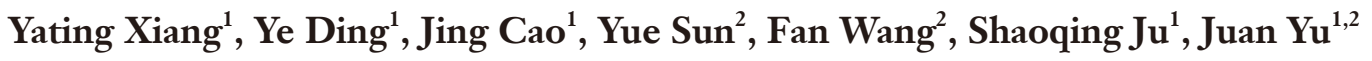 \\ ${ }^{1}$ Department of Laboratory Medicine, Affiliated Hospital of Nantong University, Nantong, China; ${ }^{2}$ Institute of Public Health, Nantong University, \\ Nantong, China \\ Contributions: (I) Conception and design: All authors; (II) Administrative support: All authors; (III) Provision of study materials or patients: None; \\ (IV) Collection and assembly of data: All authors; (V) Data analysis and interpretation: All authors; (VI) Manuscript writing: All authors; (VII) Final \\ approval of manuscript: All authors. \\ Correspondence to: Juan Yu. Department of Laboratory Medicine, Affiliated Hospital of Nantong University, 20 Xi Si Road, Nantong 226001, China. \\ Email: yujuanjs@163.com.
}

\begin{abstract}
The prevalence of antibiotic resistance is a growing worldwide problem in the control of pathogens, particularly negative bacteria that are resistant to antibiotics, Pseudomonas aeruginosa (PA) is one of these bacteria. The development of new effective antibiotics is time-consuming and costly, and the new antibiotics may become resistant again. Therefore, non-antibiotic clinical treatment for antibiotic-resistant $\mathrm{PA}$ infection is necessary and needs to be strengthened. The antibiotic resistance (AR) mechanism of PA is complex. Biofilm formation is one of the reasons why its resistance is difficult to overcome. The formation of biofilms is mainly regulated by quorum sensing (QS). QS is a mechanism by which PA increases its virulence by producing small diffusible molecules, which regulates a series of genes associated with virulence and nutrient acquisition. QS inhibitors are potions that obstruct QS systems in bacteria and destruction of virulence. This review summarizes AR mechanism of PA, Basic knowledge of QS of PA and some nonantibiotic methods for inhibiting PA, including QS inhibitors, which have potential and far-reaching significance for antibiotic-resistant PA's clinical treatment. The review helps to provide new ideas and new schemes for clinical anti-PA infection research and treatment, and has positive significance for delaying the occurrence of bacterial drug resistance and antibiotic use management.
\end{abstract}

Keywords: Pseudomonas aeruginosa; antibiotic resistance; quorum sensing (QS); QS inhibitors; other non-antibiotic approaches

Submitted Nov 11, 2020. Accepted for publication Apr 15, 2021.

doi: 10.21037/apm-20-2247

View this article at: http://dx.doi.org/10.21037/apm-20-2247

\section{Introduction}

Antimicrobial resistance (AMR) is considered an important global challenge to human health. ESKAPE pathogens (Enterococcus faecium, Acinetobacter baumannii, Staphylococcus aureus, Klebsiella pneumoniae, Pseudomonas aeruginosa, and Enterobacter spp.) are the leading cause of nosocomial infections worldwide $(1,2)$. Before all, PA has been highlighted by the World Health Organization as an important pathogen connected with AMR $(1,2)$. More than 50,000 nosocomial infections and 400 deaths related to PA are reported annually in the USA (3). Important characteristics that contribute to the PA pathogenicity are metabolic diversity, production of many virulence factors, biofilm formation, and AR (4). AR arises from the overuse of antibiotics during treatment (5). Several published studies on reducing antibiotic use or discovering new antimicrobials, such as the combination of antibiotics with non-antibiotics against clinical multidrug resistant (MDR) PA (6). PA not only has high intrinsic resistance but also can coexist with drugs as a biofilm to achieve adaptive AR (4). The formation of biofilms is mainly regulated by QS (7), QS is the principal administrative mechanism, which controls biofilm 
formation, efflux pump, secretion system, movement and AR in a group density-dependent manner. It also regulates the production of various virulence portions, such as alginate, proteases, toxins, and siderophores (8). This article summarizes PA's AR mechanism, basic knowledge about QS, and some non-antibiotic treatments against PA infections including QS inhibitors, non-quorum sensing inhibitor methods, and a combination of these 2 approaches. We present the following article in accordance with the Narrative Review reporting checklist (available at http://dx.doi.org/10.21037/apm-202247).

\section{Methods}

PubMed searches were performed using the terms: "Pseudomonas aeruginosa", "Pseudomonas aeruginosa and antibiotic resistance", and "Pseudomonas aeruginosa and non-antibiotic" for all study designs. Search results were reviewed for relevance.

\section{AR mechanism of $P A$}

AR mechanism of PA is complex, and several factors are involved (9-12): first, changes in cell membrane permeability: (I) high expression efflux pumps on the cell membrane of PA that transport drugs to the outside of cells; (II) the loss or low expression of membrane poreforming proteins: the main drug-resistant mechanism of carbapenems is the loss or decrease of outer membrane pore-forming protein OprD2. Second, the production of inactivated enzymes or modified enzymes on PA, such lactamase and aminoglycosidase. Third, changes in PA drug targets. Changes in penicillin-binding proteins lead to the resistance of penicillin drugs, and DNA enzymes II and IV structure topology changes result in drug resistance to fluoroquinolone drugs. Finally, the formation of biofilms on PA. Bacteria can survive by using biofilms to escape the body's immune system and antimicrobial agents that kill them. The AR mechanism of PA is complex (13).

\section{Basic knowledge of QS of PA:}

QS is negotiated by a solid, interconnected system consisting of the following 4 public systems: 2 LuxR/LuxI-type (LasR/ LasI and RhlR/RhlI), together with an orphan LuxR-type receptor (QscR), and the quinolone system PqsR. LasI catalyzes the production of the diffusible QS signal N-3oxododecanoyl homoserine lactone (3OC12-HSL), 3OC12-
HSL connects to its analogous receptor, the transcription factor LasR, which can then activate the appearance of multiple genes, including lasI, rblI and $r b l R$, analogously, RhlI catalyzes the generation of the diffusible beacon butyrylHSL (C4-HSL), which connects to the transcription factor RhlR, signal-bound RhlR stimulates the expression of RhlI and some genes, some of which continue to activate the LasR regulon (14-18). PqsR is unrelated to LuxR-type receptors, which is negotiated by the signal 2-heptyl-3hydroxy-4-quinolone (Pseudomonas quinolone signal, PQS) and its biosynthetic precursor 2-heptyl-4-quinolone (HHQ) $(19,20)$. The environment makes PA more pathogenic, and this process is mainly controlled by these 4 systems $(14,21)$. The characteristics of many PA virulence factors and distinct genes is principally regulated by 2 acyl-homoserine lactone (AHL) QS systems, LasI/LasR and RhII/RhlR $(8,20)$. LasR is central to the QS system (22). For example, LasR's activation can cause a cascade of reactions to turn on several PA virulence factors by activating the RhIIR and PQS systems $(14,15)$. LasR and RhlR are suppressed by QscR, which is stimulated through innate sign 3OC12-HSL $(18,21)$. PqsR system and AHL systems interact with each other $(22,23)$. The lasR gene encodes a QS regulator that is usually observed in clinical isolates with deleterious-function mutations (24). Interestingly, LasR-strains exhibites strong activity of the oxygen-sensitive transcription factor Anr in microoxic states. Mhr is an Anr-regulated microoxic hemerythrin that ties oxygen, both Anr and Mhr are crucial for the adaptation of microhypoxia, and certain genes hold unique advantages for LasR-strains in biofilms grown under normoxic conditions $(22,25)$. The development of PA in chronic infections is partly due to its tendency to lose LasR function resulting in increased microoxygenation fitness $(22,25)$. Studies show that PA LasR-strain has higher Anr activity, and the adaptive ability of Anr regulation was stronger in the microoxygenation environment, $\Delta$ lasR mutations increase the adaptability in colony biofilms grown in micro-oxygenation or oxygen atmosphere, this progression relies on Anr and Anr-regulated Mhr $(22,26)$. Surface assistance, which is essential for various group behaviors, favors more active QS responses (27). It was found that the QS main regulator LasR is upregulated during surface association, making surfaceassociated cells more susceptible to the LasR ligand 3OC12HSL (27). QS resembles to be essential for PA adaptability. For example, the toxicity of QS-null mutants is reduced in acute animal infection models. This situation exercises stress on LasR mutant cells to recover at least a portion of their QS regulon by activating the RhIIR loop $(22,23)$. 

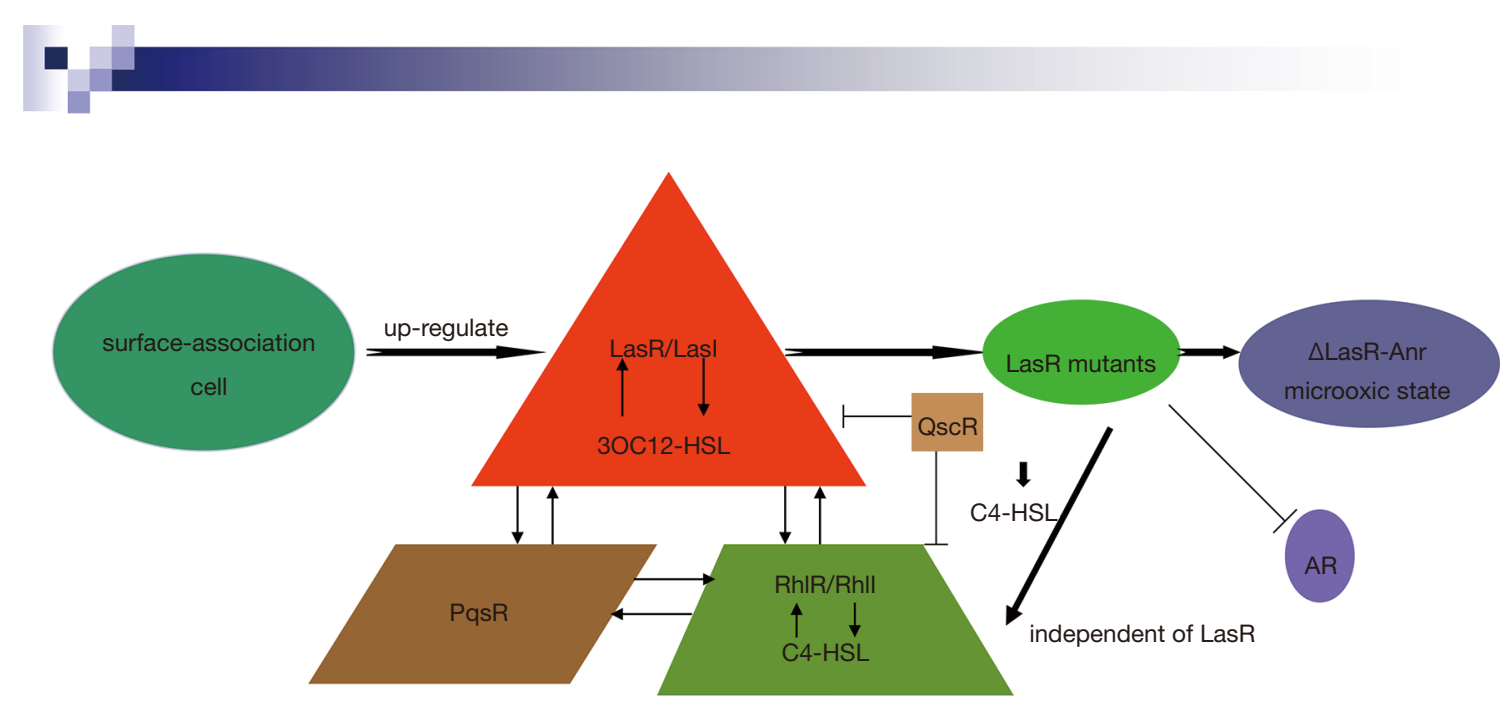

A simple schematic of the relationships between 4 systems and the relationships with the environment and antibiotic resistance; - : suppress; $\downarrow$ C4-HSL:stimulation of C4-HSL was given; AR: antibiotic resistance.

Figure 1 A simple schematic of the relationships between 4 systems and the relationships with the environment and antibiotic resistance.

Giving this condition of C4-HSL make the RhIIR way free of LasR by a surprisingly simple genetic variation, which gives rise to the RhlI-RhlR system independent of LasR and then it can continue to grow without C4-HSL (23). The study found that if the lasR mutant is selected first, this will limit AR evolution (28). Conversely, if the drug-resistant mutation is selected, the lasR mutation may not be selected in the presence of antibiotics, highlighting the importance of chance and epistatic interactions in regulating the evolution of AR (28). The clinical separation rate of MDR PA is increasing, PA has a complex QS network, which plays a vital part in its ability to adapt to multiple environments (27). The prevalence of MDR PA has developed in the past few decades and is currently $15-30 \%$ in some western European countries (29). QS inhibitors are agents that destroy the QS system in bacterial cells, reduce the production of virulence factors, and inhibit virulence without disrupting bacterial growth; therefore, resistance to these agents is not expected to be produced or developed (8) (as shown in the Figure 1).

\section{QS inhibitors}

\section{LasR antagonist V-06-018}

V-06-018 is a small abiotic particle found during high flux screening and is one of the most effective recognized LasR antagonists. The structure-activity relationship controlling
LasR antagonism by V-06-018 has been reported. V-06018 is a comparatively effective LasR antagonist in both Escherichia coli and PA LasR reporter strains and has been confirmed to inhibit virulence-related genes and phenotypes in PA (8). At the same time, V-06-018 lacks lactone parts so it is not easily hydrolyzed or enzymatically decomposed by AHL lactonases. Biochemical experiments have investigated the antagonistic mechanism of $\mathrm{V}-06-018$ and its analogs, the compound is the most effective antagonist of LasR known and is a powerful probe to characterize the mechanism of action of LuxR-type QS and can be used in general chemical and biological studies in the growing field of QS (8).

\section{Metallic oxide nanoparticles}

In recent years, advances in nanotechnology have led to an increase in their use in medicine, particularly in treating infectious diseases. Nanoparticles are transparent in the visible range and are semiconductors in nature, with great refractive index, large specific surface area and volume, high optical chemical steadiness and good environmental biocompatibility $(30,31)$. These new traits make them excellent candidates for a variety of biomedical applications. Metallic oxide nanoparticles such as zinc oxide $(\mathrm{ZnO})$ and silver oxide have a good inhibitory effect on drug-resistant strains. ZnO nanoparticles significantly downregulate QS regulatory genes' corresponding expression, lasI, lasR, 
rhlI, rhlR, pqsA and pqsR and disrupt the QS circuit and therefore repressing the production of virulence factors. $\mathrm{ZnO}$ nanoparticles are promising QS inhibitors and antiviral compounds, which can be used as adjuvant drugs for PA infections such as burns and surgical wound infections (32). Also, Chitosan significantly reduced the QSdependent phenotype and QS regulatory gene expression and inhibits the production of protease and pyocyanin in a dose-dependent manner. The preparation of chitosan$\mathrm{ZnO}$ nanocomposite enhances the antibacterial activity of chitosan (33).

\section{Drugs}

Metformin, glyceryl trinitrate, and diclofenac sodium have been found to restrain QS in the PA PAO1 strain (34-36). The activity of bacteria is relevant to their ability to adhere to tissues and form biofilms. The bacterial movement is directed by the LasI/R and RhlI/R QS systems. Sitagliptin can significantly block PA's ability to move, gather and convulse, and its movement is higher than that of metformin. To investigate the binding ability of sitagliptin to receptors LasR and rhlR, molecular docking studies were performed to attach sitagliptin to the active sites of LasR and rhlR. The high docking fraction indicated that sitagliptin has good antagonistic activity, making sitagliptin a promising antagonist that could interfere with the autoinducers' binding to their receptors. In summary, sitagliptin is a novel anti-QS drug that can be used to prevent infections caused by drug-resistant PA in diabetic patients. In non-diabetic patients, it can be used locally to heal the wound and burn infections from burns caused by PA (37). Other drugs that inhibit the QS system of PA include ibuprofen and tea polyphenols $(38,39)$, etc.

\section{Aryl bydrocarbon receptor (AbR)}

A model of host regulation by aryl hydrocarbon receptor (AhR) was studied, which relied heavily on PA's qualitative and quantitative detection. QS molecules bind to AhR and regulate its activity (40). Alterations in the expression of self-inducible factors and QS regulatory genes affect the dynamics of the bacterial community and the response of the host during the process of infection. AhR is a conserved ligand-dependent transcription factor that directly recognizes PA phenazines and plays a vital role in infection control, which binds to phenazines, mediate their degradation and regulates several host genes' expression including detoxifying enzymes, chemokines, and cytokines (40-42). Therefore, different QS molecules are related to the severity of infection. Because AhR can identify diverse levels of PA QS molecules (including Pyo or PQS) and distinguish strain-related diversity during disease, thereby regulating host responses subsequently. AhR functions as a host sensor to monitor several QS molecules and their expression forms during infection and disease, therefore, the host can adjust its protected defenses depending on the bacterial community's degree and density and the threat of infection. By monitoring communication between bacteria, AhR can sense PA communities' status during infection, enabling the host to mobilize the most suitable protection mechanisms according to the degree of danger (43).

\section{KS8 isolate}

The KS8 isolate, a marine Pseudoalteromonas spp, produces QS inhibitors that are active against PA PAO1, the supernatant of which results in an obvious decrease in biofilm biomass and total viable counts of PA during the process of biofilm formation and eradication, the extract of which can also reduce PA biofilm. The efficacy of the combination of tobramycin and KS8-derived QS inhibitors in the treatment of PA PAO1 was studied in vitro to verify further QS inhibitors' strategy combined with the sub-therapeutic concentration of traditional antibacterial drugs (44). For characterization and purification of QS inhibitors, the KS8 isolate is still an ideal candidate and has clinical relevance (44).

\section{Non-QS inhibitor methods}

\section{Antimicrobial photothermal therapy (PTT)}

PTT uses nanomaterials that are activated by absorbing specific wavelengths of near-infrared (NIR) light in the 700$1,100-\mathrm{nm}$ range, with a sufficiently long tissue penetration depth (45). Conductive polymers such as polypyrrole $(\mathrm{PPy})$ are widely used as contrast agents in optical coherence tomography because of their strong absorption of NIR light, which combines non-toxic compounds with appropriate wavelengths of light (46). As a nanomaterial, the carbon core-PPy shell nanostructure $(\mathrm{C}-\mathrm{PPy})$ is mainly responsible for its bactericide effect due to its lighttrapping properties, higher sterilization temperature and reactive oxygen species generation induction. Combining the characteristics of these 2 materials as C-PPy mixtures, composites, or complex structures may improve their 
stability, biocompatibility, NIR absorbance, and/or PPT performance. Laser exposure to the C-PPy attached to the PA surface changes the permeability of the membrane. ROS also reacts with enzymes, proteins, lipids, and other cellular components in bacterial cells, resulting in cell death. Photoablation is a new minimally invasive and inexpensive antibacterial therapy without any excessive risk to normal cells (47). Other, 50-100 nm long gold nanorods presented on heteromultivalent and 3D display of 2 types of glycomimetic polymers can prevent the colonization of the bacteria and potential the formation of the biofilm by targeting LecA and LecB lectins on PA specifically, and they have shown the photothermal characteristics of killing pathogenic cells upon NIR light irradiation (48). Photodynamic therapy (PDT) of 5-aminolevulinic acid combined with ethylenediamine tetraacetic acid disodium salt (EDTA-2Na) has a bactericidal effect on PA in vitro and promotes wound healing in mice with PA-infected cutaneous ulcers (49).

\section{Antimicrobial peptides (AMPs)}

AMPs have the advantages of broad-spectrum activity, high efficiency at low concentration, strong targeting specificity, low drug resistance tendency, and synergistic effect with traditional antibiotics (50). They are a large class of naturally occurring antimicrobials that have been identified in plants and humans in the innate immune system of several species (51-55). Cationic AMPs (CAMPs) are highly effective in killing resistant bacterial strains (56). By destroying the integrity of nucleic acids, bacterial membranes, and proteins or inhibiting intracellular function, CAMPs can show direct activity against diverse cellular targets. Diverse groups of CAMPs are effective against bacteria $(54,56,57)$. Cecropins and cecropin-derived CAMPs are antimicrobial peptides with bactericidal activity against wild-type and MDR bacteria $(57,58)$. A synthetic CAMPs derived from cecropin D-like peptide from the Galleria mellonella called $\Delta \mathrm{M} 2$ also have an antimicrobial effect on PA's clinical strains (59). A new series of biphenylglyoxamide-based small molecular AMP mimics have been identified as potential leads to treat bacterial infections, synthesized from N-sulfonylation's ring-opening reaction bearing a biphenyl backbone with a diamine (60). A Janus-type antimicrobial dressing consists of electrospun nanofiber membranes coupled with dissolvable microneedle arrays to enable effective delivery of a database-designed antimicrobial peptide to both inside and outside biofilms. This can also completely remove the
PA dual-species biofilm in an ex vivo human skin infection model (61).

\section{Antibacterial peptide nanomaterial}

Bifunctional antibacterial peptides consist of a membranelocalizing peptide and a tandem peptide. To delay and prolong the release after lung delivery, the tandem peptide is loaded into porous silicon nanoparticles (pSiNPs). The membrane-localizing peptide must be accompanied by a synthetic bacterial toxin (a toxic peptide cargo) $\mathrm{D}[\mathrm{KLAKLAK}] 2, \mathrm{D}[\mathrm{KLAKLAK}] 2$ is independent of its stereochemistry and it was synthesized with $\mathrm{D}$-amino acids to limit proteolytic degradation. It was called $\mathrm{dKK}$, which has no activity against PA at the concentrations studied (62-64). Lactoferrin-dkk (lact-dkk) was found to be the bestperforming tandem peptide. It is known that lactoferrin peptide, KCFQWQRNMRKVRGPPVSCIKR, interacts with bacterial membranes (65). LACT-dKK kills bacteria at a submicromolar concentration $\left(0.42 \times 10^{-6} \mathrm{~mol}\right)$. The killing mediated by peptide series exceeded the expected additive reaction of 2 single peptide domains, indicating the synergistic effect between the 2 peptide domains in the tandem peptide structure. The best performing peptide was formulated into a biodegradable pSiNP nanoparticle, and after delivery to the lung in the mouse model of pulmonary infection, bacterial titers decreased significantly. Clinical isolates from human pulmonary infections are susceptible to peptide treatment, suggesting that the method could be applied to other PA strains (66).

\section{Mucin glycans}

A kind of slimy, watery mucus gel sets the first line of defense and simultaneously houses the trillions of microbes, and mucus gel was found in all wet epithelial cells in the body, the eyes, lungs, and gastrointestinal and urogenital tracts. In healthy mucus, microbes rarely cause infections. Studies have ascertained that exposure to mucus induces the downregulation of virulence genes included in QS, siderophore biosynthesis, toxin secretion, and the rapid decomposition of biofilm using a 3D laboratory model of natural mucus and PA. This phenotypic switch is triggered by mucins, polymers that are tightly attached to the O-chain-glycan to form a $3 \mathrm{D}$ scaffold within the mucus, which work at different scales to inhibit different toxic pathways, promote plankton lifestyle, reduce toxicity to human epithelial cells in vitro, and attenuate infection 
in a porcine burn model. It was found that mucin-related glycans mediated the interaction with PA. Even at relatively low concentrations, bacterial phenotypes can be effectively regulated. Complex O-linked glycans are the main regulatory component of mucins. This regulatory function may depend on glycan's complexity, as monosaccharides do not attenuate the virulence of PA, indicating that the complex arrangement and special stereochemistry of these sugar residues are crucial to their function as regulatory signals (67).

\section{Cold atmospheric plasma (CAP)}

CAP is a low-temperature ionized or partially ionized gas (approximately $20-40{ }^{\circ} \mathrm{C}$ ) that makes it suitable for use in living cells and tissues $(68,69)$. Recent studies have shown that CAP's antibacterial action is mainly the production of long- and short-lived active substances (ROS and reactive nitrogen species) (70-74). CAP treatment can be divided into direct treatment and activated-liquid treatment (71). Direct therapy is done using devices that directly touch cells or the body. CAP device-activated fluids, or cap-activated fluids, have strong therapeutic potential similar to the effects of direct CAP therapy (73). Studies found that CAP treatment increased intracellular ROS levels, which was even greater during direct treatment. Similar increases in intracellular ROS levels have also been reported in cancer cell lines after CAP treatment due to long-lived and shortlived species' production, particularly short-lived species. CAP treatments rapidly eliminate single-species and mixed-species biofilms and can lead to loss of membrane integrity and increased cell permeability. However, with the accelerated development of bacterial resistance, it is important to study CAP treatment's effect on MDR bacteria. MDR PA strains isolated from hospitalized patients are more consistent with current and future clinical conditions. The results showed that CAP treatment was effective against strains resistant to multiple antibiotics, indicating its prospect in treating drug-resistant, Gramnegative bacteria (75).

\section{Combination therapy}

\section{Antimicrobial PDT (APDT)}

APDT is a medical treatment consisting of visible light and non-toxic photoactivable dyes or photosensitizers (PS) in the presence of oxygen. When PS absorbs photons of the right wavelength, it leads to cytotoxic ROS formation, which leads to cell death (76,77). Curcumin-induced APDT inhibits biofilm formation more effectively through ROS generation, especially type II photochemical reactions. The mechanism of biofilm inhibition is to suppress PA's QS system to remove extracellular polymeric substances (EPS). EPS are an important component of biofilm, which provides the mechanical stability of biofilm against foreign inclusion. Curcumin is widely combined with the QS system of photoinhibition. Curcumin-mediated APDT may be a potential therapy for controlling the biofilm-mediated infection. Bacteria can also develop antibiotic resistance and the host immune system through the intercellular signaling particles produced by bacteria in biofilms. The utilization of PS is important for the effectiveness of APDT. Being an amphiphilic PS and photothermal agent, indocyanine green (ICG) is safe for human body and has a NIR (600$950 \mathrm{~nm}$ ) excitation wavelength, further promoting the photobiological effect of deep tissue to achieve bactericidal effect $(78,79)$. A variety of micro-organisms causes burn wound infection, and the regulation of multiple colonization and virulence factors in intercellular communication and micro-organisms is regulated by the QS system. APDT is an effective treatment option for infected ulcers and can reduce antimicrobial use and resistance induction. As an anionic medium, ICG can effectively produce ROS required by APDT under NIR laser irradiation. ICG-APDT could downregulate the expression levels of abaI, agrA, and lasI. Amid the reduction of these gene expressions, biofilm formation is also disrupted. ICG-APDT effectively induces the production of lipid peroxidation, superoxide free radicals, and intracellular ROS in multispecies biofilm culture. Simultaneously, it significantly reduces the number of bacterial cells and the expression level of QS genes of major pathogens on burn wounds. APDT with ICG combined with antibacterial, anti-biofilm, and gene expression inhibitors effectively treat multispecies bacterial biofilms correlated with burn and wound infection $(80,81)$.

\section{Bacteriophage therapy}

Phages can destroy biofilm formation of PA by blocking QS activity and can provide a possible alternative method to reduce the colonization of the endotracheal tube surface by bacteria. Also, phages in combination with other compounds (such as nanoparticles, enzymes, and natural products) are more effective than using them alone $(82,83)$. 


\section{Discussion and summary}

$\mathrm{AR}$ mechanism of $\mathrm{PA}$ is complex, and it takes time and effort to explore new antibiotics, and the use of new antibiotics is easy to make PA resistant again $(5,84)$. Therefore, PA's non-antibiotic treatment is important, and it is speculated that there will be more and more non-antibiotic treatments in the future. The AR of PA is closely related to the QS system. There is a complicated and subtle relationship between diverse QS of PA, and it is a hierarchical and orderly relationship (85). The inhibition of QS is a bacterial communication model that relies on a chemical signal exchange and has been shown to reduce the virulence phenotype of various human pathogenic bacteria, and the use of QS inhibitors does not make the bacteria resistant. Therefore, as a prospective antimicrobial approach, QS inhibitors are of great concern.

Although various non-antibiotic treatments for PA have emerged, most are limited to in vitro or mouse tests. They are expected to be formally used in clinical trials, bringing new hope for the treatment of drug-resistant bacteria. Various therapeutic combinations are also expected to treat the resistance problem of PA.

\section{Acknowledgments}

The manuscript was edited for proper English language, grammar, punctuation, spelling, and overall style by one or more of the English speaking editors at AME Publishing Company.

Funding: This work was supported by the Science and Technology Project of Nantong city (JC2018067).

\section{Footnote}

Reporting Checklist: The authors have completed the Narrative Review reporting checklist. Available at http:// dx.doi.org/10.21037/apm-20-2247

Conflicts of Interest: All authors have completed the ICMJE uniform disclosure form (available at http://dx.doi. org/10.21037/apm-20-2247) and report that this work was supported by the Science and Technology Project of Nantong city (JC2018067). The authors have no other conflicts of interest to declare.

Ethical Statement: The authors are accountable for all aspects of the work in ensuring that questions related to the accuracy or integrity of any part of the work are appropriately investigated and resolved.

Open Access Statement: This is an Open Access article distributed in accordance with the Creative Commons Attribution-NonCommercial-NoDerivs 4.0 International License (CC BY-NC-ND 4.0), which permits the noncommercial replication and distribution of the article with the strict proviso that no changes or edits are made and the original work is properly cited (including links to both the formal publication through the relevant DOI and the license). See: https://creativecommons.org/licenses/by-nc-nd/4.0/.

\section{References}

1. Cazares A, Moore MP, Hall JPJ, et al. A megaplasmid family driving dissemination of multidrug resistance in Pseudomonas. Nat Commun 2020;11:1370.

2. Surivet JP, Panchaud P, Speckli JL, et al. Discovery of Novel Inhibitors of LpxC Displaying Potent In Vitro Activity against Gram-Negative Bacteria. J Med Chem 2020;63:66-87.

3. Azam MW, Khan AU. Updates on the pathogenicity status of Pseudomonas aeruginosa. Drug Discov Today 2019;24:350-9.

4. Pang Z, Raudonis R, Glick BR, et al. Antibiotic resistance in Pseudomonas aeruginosa: mechanisms and alternative therapeutic strategies. Biotechnol Adv 2019;37:177-92.

5. Zhu K, Chen S, Sysoeva TA, et al. Universal antibiotic tolerance arising from antibiotic-triggered accumulation of pyocyanin in Pseudomonas aeruginosa. PLoS Biol 2019;17:e3000573.

6. Vipin C, Saptami K, Fida F, et al. Potential synergistic activity of quercetin with antibiotics against multidrugresistant clinical strains of Pseudomonas aeruginosa. PLoS One 2020;15:e241304.

7. Başaran TI, Berber D, Gökalsın B, et al. Extremophilic Natrinema versiforme Against Pseudomonas aeruginosa Quorum Sensing and Biofilm. Front Microbiol 2020;11:79.

8. Husain FM, Ansari AA, Khan A, et al. Mitigation of acyl-homoserine lactone (AHL) based bacterial quorum sensing, virulence functions, and biofilm formation by yttrium oxide core/shell nanospheres: Novel approach to combat drug resistance. Scientific Reports 2019;9:18476.

9. Ahmed MN, Abdelsamad A, Wassermann T, et al. The evolutionary trajectories of $\mathrm{P}$. aeruginosa in biofilm and planktonic growth modes exposed to ciprofloxacin: beyond selection of antibiotic resistance. NPJ. Biofilms 
Microbiomes 2020;6:28.

10. $\mathrm{Lu} \mathrm{Y,} \mathrm{Li} \mathrm{H,} \mathrm{Pu} \mathrm{J,} \mathrm{et} \mathrm{al.} \mathrm{Identification} \mathrm{of} \mathrm{a} \mathrm{novel} \mathrm{RhlI/}$ R-PrrH-LasI/Phzc/PhzD signaling cascade and its implication in P. aeruginosa virulence. Emerg Microbes Infect 2019;8:1658-67.

11. Geisinger E, Isberg RR. Interplay Between Antibiotic Resistance and Virulence During Disease Promoted by Multidrug-Resistant Bacteria. J Infect Dis 2017;215:S9-S17.

12. Cunrath O, Meinel DM, Maturana P, et al. Quantitative contribution of efflux to multi-drug resistance of clinical Escherichia coli and Pseudomonas aeruginosa strains. EBioMedicine 2019;41:479-87.

13. Olivares E, Badel-Berchoux S, Provot C, et al. Pseudomonas aeruginosa Clinical Impact of Antibiotics for the Treatment of Biofilm Infections. Front Microbiol 2020;10:2894.

14. Lee J, Zhang L. The hierarchy quorum sensing network in Pseudomonas aeruginosa. Protein Cell 2015;6:26-41.

15. Seed PC, Passador L, Iglewski BH. Activation of the Pseudomonas aeruginosa lasI gene by LasR and the Pseudomonas autoinducer PAI: an autoinduction regulatory hierarchy. J Bacteriol 1995;177:654-9.

16. Papenfort K, Bassler BL. Quorum sensing signal-response systems in Gram-negative bacteria. Nat Rev Microbiol 2016;14:576-88.

17. Zhang B, Ren L, Xu D, et al. Directed evolution of RhlI to generate new and increased quorum sensing signal molecule catalytic activities. Enzyme Microb Technol 2020;134:109475.

18. Manson DE, O'Reilly MC, Nyffeler KE, et al. Design, synthesis, and biochemical characterization of non-native antagonists of the Pseudomonas aeruginosa quorum sensing receptor LasR with nanomolar IC50 values. ACS. Infect Dis 2020;6:649-61.

19. Chen R, Déziel E, Groleau MC, et al. Social cheating in a Pseudomonas aeruginosa quorum-sensing variant. Proc Natl Acad Sci U S A 2019;116:7021-6.

20. El-Mowafy SA, Shaaban MI, Abd El Galil KH. Sodium ascorbate as a quorum sensing inhibitor of $\mathrm{P}$ seudomonas aeruginosa. J Appl Microbiol 2014;117:1388.

21. Déziel E, Lépine F, Milot S, et al. Analysis of Pseudomonas aeruginosa 4-hydroxy-2-alkylquinolines (HAQs) reveals a role for 4-hydroxy-2-heptylquinolinein cellto-cell communication. Proc Natl Acad Sci U S A 2004;101:1339-44.

22. Clay ME, Hammond JH, Zhong F, et al. Pseudomonas aeruginosa las R mutant fitness in microoxia is supported by an Anr-regulated oxygen-binding hemerythrin. Proc Natl Acad Sci U S A 2020;117:3167-73.
23. Kostylev M, Kim DY, Smalley NE, et al. Evolution of the Pseudomonas aeruginosa quorum-sensing hierarchy. Proc Natl Acad Sci U S A 2019;116:7027-32.

24. Hammond JH, Hebert WP, Naimie A, et al. Environmentally endemic Pseudomonas aeruginosa strains with mutations in lasR are associated with increased disease severity in corneal ulcers. 2016;mSphere. 1.

25. Hammond JH, Dolben EF, Smith TJ, et al. Links between Anr and quorum sensing in Pseudomonas aeruginosa biofilms. J Bacteriol 2015;197:2810-20.

26. Højberg O, Schnider U, Winteler HV, et al. Oxygensensing reporter strain of Pseudomonas fluorescens for monitoring the distribution of low-oxygen habitats in soil. Appl Environ Microbiol 1999;65:4085-93.

27. Chuang SK, Vrla GD, Fröhlich KS, et al. Surface association sensitizes Pseudomonas aeruginosa to quorum sensing. Nat Commun 2019;10:4118.

28. Hernando-Amado S, Sanz-García F, Martínez JL. Antibiotic Resistance Evolution Is Contingent on the Quorum-Sensing Response in Pseudomonas aeruginosa. Mol Biol Evol 2019;36:2238-51.

29. Del Barrio-Tofiño E, Zamorano L, Cortes-Lara S, et al. Spanish nationwide survey on Pseudomonas aeruginosa antimicrobial resistance mechanisms and epidemiology. J Antimicrob Chemother 2019;74:1825-35.

30. Aysa NH, Salman HD. Antibacterial activity of modified zinc oxide nanoparticles against Ps. aeruginosa isolates of burn infections. World Scientific News 2016;33:1-14.

31. Pati R, Mehta RK, Mohanty S, et al. Topical application of zinc oxide nanoparticles reduces bacterial skin infection in mice and exhibits antibacterial activity by inducing oxidative stress response and cell membrane disintegration in macrophages. Nanomedicine 2014;10:1195-208.

32. Saleh MM, Sadeq RA, Latif HKA, et al. Zinc oxide nanoparticles inhibits quorum sensing and virulence in Pseudomonas aeruginosa. Afr Health Sci 2019;19:2043-55.

33. Msem B, Riad OKM, Taher FA, et al. Chitosan and chitosan-zinc oxide nanocomposite inhibit expression of LasI and RhlI genes and quorum sensing dependent virulence factors of Pseudomonas aeruginosa. Int J Biol Macromol 2020;149:1109-17.

34. Abbas HA. Inhibition of virulence factors of Pseudomonas aeruginosa by diclofenac sodium. Roum Arch Microbiol Immunol 2015;74:79-85.

35. Abbas HA, Shaldam MA. Glyceryl trinitrate is a novel inhibitor of quorum sensing in Pseudomonas aeruginosa. Afr Health Sci 2016;16:1109-17.

36. Abbas HA, Elsherbini AM, Shaldam MA. Repurposing 
metformin as a quorum sensing inhibitor in Pseudomonas aeruginosa. Afr Health Sci 2017;17:808-19.

37. Abbas HA, Shaldam MA, Eldamasi D. Curtailing Quorum Sensing in Pseudomonas aeruginosa by Sitagliptin. Curr Microbiol 2020;77:1051-60.

38. Dai L, Wu TQ, Xiong YS, et al. Ibuprofen-mediated potential inhibition of biofilm development and quorum sensing in Pseudomonas aeruginosa. Life Sci 2019;237:116947.

39. Yin H, Deng Y, Wang H, et al. Tea polyphenols as an antivirulence compound Disrupt Quorum-Sensing Regulated Pathogenicity of Pseudomonas aeruginosa. Sci Rep 2015;5:16158.

40. Moura-Alves P, Fae K, Houthuys E, et al. AhR sensing of bacterial pigments regulates antibacterial defence. Nature 2014;512:387-92.

41. Dietrich LE, Price-Whelan A, Petersen A, et al. The phenazine pyocyanin is a terminalm signalling factor in the quorum sensing network of Pseudomonas aeruginosa. Mol Microbiol 2006;61:1308-21.

42. Jimenez PN, Koch G, Thompson JA, et al. The multiple signaling systems regulating virulence in Pseudomonas aeruginosa. Microbiol Mol Biol Rev 2012;76:46-65.

43. Moura-Alves P, Puyskens A, Stinn A, et al. Host monitoring of quorum sensing during Pseudomonas aeruginosa infection. Science 2019;366:eaaw1629.

44. Alessandro Busetti, George Shaw. Marine-Derived Quorum-Sensing Inhibitory Activities Enhance the Antibacterial Efficacy of Tobramycin against Pseudomonas aeruginosa. Mar Drugs 2014;13:1.

45. Henderson TA, Morries LD. Near-infrared photonic energy penetration: can infrared phototherapy effectively reach the human brain? Neuropsychiatr Dis Treat 2015;11:2191-208.

46. Au KM, Lu Z, Matcher SJ, et al. Polypyrrole nanoparticles: a potential optical coherence tomography contrast agent for cancer imaging. Adv Mater 2011;23:5792-5.

47. Behzadpour N, Sattarahmady N, Akbari N. Antimicrobial Photothermal Treatment of Pseudomonas Aeruginosa by a Carbon Nanoparticles-Polypyrrole Nanocomposite. J Biomed Phys Eng 2019;9:661-72.

48. Zhao Y, Guo Q, Dai X, et al. A Biomimetic NonAntibiotic Approach to Eradicate Drug-Resistant Infections. Adv Mater 2019;31:e1806024.

49. Katayama B, Ozawa T, Morimoto K, et al. Enhanced sterilization and healing of cutaneous pseudomonas infection using 5-aminolevulinic acid as aphotosensitizer with 410-nm LED light. J Dermatol Sci 2018;90:323-31.
50. Sinha R, Shukla P. Antimicrobial Peptides: Recent Insights on Biotechnological Interventions and Future Perspectives. Protein Pept Lett 2019;26:79-87.

51. Aslam B, Wang W, Arshad MI, et al. Antibiotic resistance: A rundown of a global crisis. Infect Drug Resist 2018;11:1645-58.

52. Wang S, Zeng X, Yang Q, et al. Antimicrobial peptides as potential alternatives to antibiotics in food animal industry. Int J Mol Sci 2016;17:603.

53. Reddy KVR, Yedery RD, Aranha C. Antimicrobial peptides: Premises and promises. Int J Antimicrob Agents 2004;24:536-47.

54. Fry, DE. Antimicrobial peptides. Surg Infect (Larchmt) 2018;19:804-11.

55. Guaní-Guerra E, Santos-Mendoza T, Lugo-Reyes SO, et al. Antimicrobial peptides: General overview and clinical implications in human health and disease. Clin Immunol 2010;135:1-11.

56. Geitani R, Ayoub Moubareck C, Touqui L, et al. Cationic antimicrobial peptides: Alternatives and/or adjuvants to antibiotics active against methicillin-resistant Staphylococcus aureus and multidrug-resistant Pseudomonas aeruginosa. BMC Microbiol 2019;19:54.

57. Ocampo-Ibáñez ID, Liscano Y, Rivera-Sánchez SP, et al. A Novel Cecropin d-Derived Short Cationic Antimicrobial Peptide Exhibits Antibacterial Activity Against Wild-Type and Multidrug-Resistant Strains of Klebsiella pneumoniae and Pseudomonas aeruginosa. Evol Bioinform Online 2020;16:1176934320936266.

58. Brady D, Grapputo A, Romoli O, et al. Insect cecropins, antimicrobial peptides with potential therapeutic applications. Int J Mol Sci 2019;20:5862.

59. Rivera-Sánchez SP, Agudelo-Gongora HA, Onate-Garzon $\mathrm{J}$, et al. Antibacterial Activity of a Cationic Antimicrobial Peptide against Multidrug-Resistant Gram-Negative Clinical Isolates and Their Potential Molecular Targets. Molecules 2020;25:5035.

60. Yu TT, Kuppusamy R, Yasir M, et al. Design, Synthesis and Biological Evaluation of Biphenylglyoxamide-

Based Small Molecular Antimicrobial Peptide Mimics as Antibacterial Agents. Int J Mol Sci 2020;21:6789.

61. Su Y, Mainardi VL, Wang, H, et al. Dissolvable Microneedles Coupled with Nanofiber Dressings Eradicate Biofilms via Effectively Delivering a Database-Designed Antimicrobial Peptide. ACS Nano 2020;14:11775-86.

62. Javadpour MM, Juban MM, Lo WC, et al. De novo antimicrobial peptides with low mammalian cell toxicity. J Med Chem 1996;39:3107-13. 
63. McGrath DM, Barbu EM, Driessen WH, et al. Mechanism of action and initial evaluation of a membrane active all-D-enantiomer antimicrobial peptidomimetic. Proc Natl Acad Sci U S A 2013;110:3477-82.

64. Ellerby HM, Arap W, Ellerby LM, et al. Anti-cancer activity of targeted pro-apoptotic peptides. Nat Med 1999;5:1032-8.

65. Yamauchi K, Tomita M, Giehl TJ, et al. Antibacterial activity of lactoferrin and a pepsin-derived lactoferrin peptide fragment. Infect Immun 1993;61:719-28.

66. Kwon EJ, Skalak M, Bertucci A, et al. Porous Silicon Nanoparticle Delivery of Tandem Peptide Anti-Infectives for the Treatment of Pseudomonas aeruginosa Lung Infections. Adv Mater 2017;29.

67. Wheeler KM, Carcamo-Oyarce G, Turner BS, et al. Mucin glycans attenuate the virulence of Pseudomonas aeruginosa in infection. Nat Microbiol 2019;4:2146-54.

68. Weiss M, Daeschlein G, Kramer A, et al. Virucide properties of cold atmospheric plasma for future clinical applications. J Med Virol 2017;89:952-9.

69. Puligundla P, Mok C. Inactivation of spores by nonthermal plasmas. World J Microbiol Biotechnol 2018;34:143.

70. Gilmore BF, Flynn PB, O’brien S, et al. Cold plasmas for biofilm control: opportunities and challenges. Trends Biotechnol 2018;36:627-38.

71. Kaushik NK, Ghimire B, Li Y, et al. Biological and medical applications of plasma-activated media, water and solutions. Biol Chem 2018;400:39-62.

72. Kondeti VS, Phan CQ, Wende K, et al. Long-lived and short-lived reactive species produced by a cold atmospheric pressure plasma jet for the inactivation of Pseudomonas aeruginosa and Staphylococcus aureus. Free Radic Biol Med 2018;124:275-87.

73. Nguyen L, Lu P, Boehm D et al. Cold atmospheric plasma is a viable solution for treating orthopedic infection: a review. Biol Chem 2018;400:77-86.

74. Privat-Maldonado A, Gorbanev Y, Dewilde S, et al. Reduction of human glioblastoma spheroids using cold atmospheric plasma: the combined effect of short- and long-lived reactive species. Cancers (Basel) 2018;10:394.

75. Wang L, Xia C, Guo Y, et al. Bactericidal efficacy of cold atmospheric plasma treatment against multidrug-resistant Pseudomonas aeruginosa. Future Microbiol 2020;15:115-25.

76. Vaid D, Shah N, Kothari D, et al. Additive effect of photoactivated disinfection on the antibacterial activity of QMix 2in1 against 6-week Enterococcus faecalis biofilms: an in vitro study. J Conserv Dent 2017;20:41-5.
77. Pourhajibagher M, Bahador A. Gene expression profiling of fimA gene encoding fimbriae among clinical isolates of Porphyromonas gingivalis in response to photo-activated disinfection therapy. Photodiagnosis Photodyn Ther 2017;20:1-5.

78. Pourhajibagher M, Chiniforush N, Ghorbanzadeh R, et al. Photo-activated disinfection based on indocyanine green against cell viability and biofilm formation of Porphyromonas gingivalis. Photodiagnosis Photodyn Ther 2017;17:61-4.

79. Pourhajibagher M, Chiniforush N, Shahabi S, et al. Monitoring gene expression of rcpA from Aggregatibacter acti-nomycetemcomitans versus antimicrobial photodynamic therapy by relative quantitative real-time PCR. Photodiagnosis Photodyn. Ther 2017;19:51-5.

80. Pourhajibagher M, Mahmoudi H, Rezaei-Soufi L, et al. Potentiation effects of antimicrobial photodynamic therapy on quorum sensing genes expression: A promising treatment for multi-species bacterial biofilms in burn wound infections. Photodiagnosis. Photodyn Ther 2020;30:101717.

81. Abdulrahman H, Misba L, Ahmad S, et al. Curcumin induced photodynamic therapy mediated suppression of quorum sensing pathway of Pseudomonas aeruginosa: An approach to inhibit biofilm in vitro. Photodiagnosis Photodyn Ther 2020;30:101645.

82. Chegini Z, Khoshbayan A, Taati Moghadam M, et al. Bacteriophage therapy against Pseudomonas aeruginosa biofilms: a review. Ann Clin Microbiol Antimicrob 2020;19:45.

83. Oliveira VC, Bim FL, Monteiro RM, et al. Identification and Characterization of New Bacteriophages to Control Multidrug-Resistant Pseudomonas aeruginosa Biofilm on Endotracheal Tubes. Front Microbiol 2020;11:580779.

84. Botelho J, Grosso F, Peixe L. Antibiotic resistance in Pseudomonas aeruginosa - Mechanisms, epidemiology and evolution. Drug Resist Updat 2019;44:100640.

85. Chang CY, Krishnan T, Wang H, et al. Non-antibiotic quorum sensing inhibitors acting against $\mathrm{N}$-acyl homoserine lactone synthase as druggable target. Sci Rep 2014;4:7245.

Cite this article as: Xiang Y, Ding Y, Cao J, Sun Y, Wang F, Ju $\mathrm{S}$, Yu J. Non-antibiotic methods against Pseudomonas aeruginosa include QS inhibitors: a narrative review. Ann Palliat Med 2021;10(6):6926-6935. doi: 10.21037/apm-20-2247 